\title{
Reducing False Triggering Caused by Irrelevant Mental Activities in Brain-Computer Interface Based on Motor Imagery
}

\section{Lujia Zhou}

Tianjin University https://orcid.org/0000-0002-3197-5746

\section{Xuewen Tao}

Tianjin University

\section{Feng $\mathrm{He}$}

Tianjin University

\section{Peng Zhou}

Tianjin University

\section{Hongzhi Qi ( $\square$ qhz@tju.edu.cn )}

Tianjin University https://orcid.org/0000-0001-5719-2718

\section{Research}

Keywords: brain-computer interface, false triggering, motor imagery, steady-state somatosensory evoked potential, task specificity

Posted Date: October 13th, 2020

DOl: https://doi.org/10.21203/rs.3.rs-89367/v1

License: (1) This work is licensed under a Creative Commons Attribution 4.0 International License. Read Full License

Version of Record: A version of this preprint was published at IEEE Journal of Biomedical and Health Informatics on September 1st, 2021. See the published version at https://doi.org/10.1109/JBHI.2021.3066610. 


\title{
Reducing false triggering caused by irrelevant mental activities in brain-computer interface based on motor imagery
}

\author{
Lujia Zhou ${ }^{1}$, Xuewen $\mathrm{Tao}^{1}$, Feng He ${ }^{1,2}$, Peng Zhou ${ }^{1,2}$, and Hongzhi Qi ${ }^{1,2 *}$
}

\begin{abstract}
Background: In recent years, the brain-computer interface (BCI) based on motor imagery (MI) has been considered as a potential post-stroke rehabilitation technology. However, the recognition of MI relies on the event-related desynchronization (ERD) feature, which has poor task specificity. Further, there is the problem of false triggering (irrelevant mental activities recognized as the MI of the target limb).

Methods: In this paper, we discuss the feasibility of reducing the false triggering rate using a novel paradigm, in which the steadystate somatosensory evoked potential (SSSEP) is combined with the MI (MI-SSSEP). Data from the target (right hand MI) and nontarget task (rest) were used to establish the recognition model, and three kinds of interference tasks were used to test the false triggering performance. In the MI-SSSEP paradigm, ERD and SSSEP features modulated by MI could be used for recognition, while in the MI paradigm, only ERD features could be used.

Results: The results showed that the false triggering rate of interference tasks with SSSEP features was reduced to 29.3\%, which was far lower than the 55.5\% seen under the MI paradigm with ERD features. Moreover, in the MI-SSSEP paradigm, the recognition rate of the target and nontarget task was also significantly improved. Further analysis showed that the specificity of SSSEP was significantly higher than that of ERD $(\mathrm{p}<0.05)$, but the sensitivity was not significantly different.
\end{abstract}

Conclusions: These results indicated that SSSEP modulated by MI could more specifically decode the target task MI, and thereby may have potential in achieving more accurate rehabilitation training.

Keywords: brain-computer interface, false triggering, motor imagery, steady-state somatosensory evoked potential, task specificity

\section{Background}

Brain-computer interface (BCI) systems based on motor imagery (MI) can decode the motion intention of the user using electroencephalography (EEG) signals [1]. In clinical applications, patients with intact brains but severely impaired motor function, resulting from spinal cord injury (SCI) [2] and amyotrophic lateral sclerosis (ALS) [3], could use MI-BCI directly to control external devices. Recent studies have found that the MI-BCI could also be used in brain rehabilitation to help stroke patients [4], [5]. This new treatment has achieved good efficacy in some reports [6], [7], which fully demonstrates the potential of MI-BCI in the field of post-stroke rehabilitation.

Different limb movements correspond to different activation areas in the sensorimotor cortex [8]. It has been confirmed that MI and motor execution (ME) have similar neural activities in the brain [9], [10]. Therefore, by decoding the EEG signals generated by MI, we could infer the actions users want to perform and thus control the external devices [11]. Previous studies have found that MI could induce event-related desynchronization (ERD) in a similar fashion to ME. The characteristics of ERD are an energy decrease in alpha (8-13

\footnotetext{
*Correspondence: qhz@tju.edu.cn.

${ }^{1}$ Department of Biomedical Engineering, College of Precision Instrument and Optoelectronics Engineering, Tianjin University, Tianjin, China.
}

$\mathrm{Hz})$ and beta (13-30 Hz) bands [12], [13]. Different imaginary tasks will induce different neural activities in sensorimotor areas. That is, there are differences in the location where the ERD occurs, by which the specific imaginary task can be identified [14], [15]. Spatial filtering methods such as the common spatial pattern (CSP) algorithm [16] are generally used to strengthen and extract ERD features, and then machine learning methods such as support vector machine (SVM) [17] are generally used to establish a classifier to identify which imaginary task occurred.

As described above, there are two primary characteristics of MI-BCI systems. First, the MI-BCI system assumes that the brain will only perform the few tasks that are accurately presented. Second, the system judges whether an imaginary task is performed by determining whether ERD is induced in the corresponding region of the brain. Generally, MI-BCI used for post-stroke rehabilitation presupposes that the brain will only perform two imaginary tasks (affected limb movement and rest). After decoding the motor intention of the affected limb, the system drives the peripheral equipment to pull the limb for passive movement, which constructs a closed-loop between

\footnotetext{
${ }^{2}$ Academy of Medical Engineering and Translational Medicine, Tianjin University, Tianjin, China.
} 
motion intention and affected limb movement. Therefore, this type of rehabilitation treatment can potentially form a better neuroinductive effect and subsequently achieve better efficacy [18], [19].

However, the MI-BCI system could not directly identify the target task MI, but it could distinguish the target task from other imaginary tasks using specific differences. The decoding of MI mainly depends on the ERD feature in the corresponding brain regions. Therefore, in the imaginary recognition of left/right hand, if ERD features in the right sensorimotor areas are detected, the BCI system will infer that the subject is performing left hand MI, and vice versa. Supposing that the right hand is the affected limb, the MI-BCI judges whether the stroke patient performs affected limb MI only if there is sufficient ERD in the left sensorimotor cortex. Nevertheless, ERD is a common phenomenon in cerebral activities. Not only could the affected limb MI induce ERD (mainly in the contralateral cerebral cortex), but many other mental activities (such as learning, memory, and even the MI or ME of the contralateral limb) could also induce ERD [20]. Although there are some differences in the intensity and spatial distribution of the induced ERD in these different tasks (because of the low signal-to-noise ratio and diffuse spatial distribution of EEG), it is difficult for MI-BCI systems to judge whether the ERD detected in the contralateral cortex is induced only by the affected limb MI [21]. The phenomenon described above leads to false triggering in MI-BCI systems. This is because MI-BCI mistakes some irrelevant mental activities for the target task (affected limb MI), so that drives peripheral equipment to pull the affected limb without the target task MI. For the passive movement driven by irrelevant mental activities, the positive effect on the rehabilitation of the damaged brain will be minimal, and there may also be a wrong neural induction effect. So it is clear that the ideal MI-BCI used for post-stroke rehabilitation should avoid such false triggering as much as possible.

However, as far as we know, there are few reports on the problem of false triggering in MI-BCI, and few papers have explored methods to reduce false triggering. As mentioned above, the problem of false triggering is mainly caused by the low task specificity of ERD features, as many irrelevant tasks can also induce ERD in the target cerebral area. Therefore, the use of EEG features with greater task specificity is a proper way to suppress false triggering. Recently, a novel paradigm with steady-state somatosensory evoked potential (SSSEP) modulated by MI has received increasing attention in MI decoding [22]. Studies have shown that SSSEP induced by somatosensory stimulation, which is applied to the target limb at a specific frequency, could be influenced by the same limb MI, thus forming a new EEG feature associated with motor consciousness [23]. MI decoding using the MI-SSSEP feature has shown many advantages, such as significantly improving the recognition rate of left/right hand MI [24] and increasing the recognition rate of adjacent joints in unilateral limb [25]. This feature comes from two neural activities: motor intention and somatosensory response to the same limb, which theoretically should have better task specificity, and thus, could be better distinguished from other mental tasks.

Therefore, the underlying assumption in this paper is that the MI-SSSEP feature has better task specificity, which can effectively reduce the false triggering in MI-BCI systems. Based on this assumption, a comparison experiment between the MI and MI-SSSEP paradigm was designed to analyze the decoding ability of the target task and false triggering of interference tasks in the hybrid (MI-SSSEP) paradigm.

\section{Methods}

\section{Subjects}

A total of 13 healthy subjects ( 7 males and 6 females, righthanded, aged between 21 and 25 years) were involved in the experiment.

\section{Experimental Paradigm}

In this paper, a comparison experiment between the MI and MISSSEP paradigm was designed. The difference between the two paradigms is that subjects received electrical stimulation at a specific frequency to induce SSSEP when performing the task under a hybrid paradigm.

Previous experiments have shown that the stimulation frequency at $31 \mathrm{~Hz}$ can induce clear SSSEP in the corresponding cerebral region [24]. Therefore, under the hybrid paradigm, stimulation was applied to the right median nerve with biphasic current pulses of $200 \mu$ s duration at $31 \mathrm{~Hz}$ [26]. Two electrode patches, which were about $4 \mathrm{~cm}$ apart, formed a loop with the electrical stimulator. The amplitude of electrical stimulation was adjusted to produce the slight quivering of the subjects' fingers, and varied from 1 to $5 \mathrm{~mA}$ for the whole experiment. Moreover, none of the subjects felt a sensation of pain. Before the formal experiment, an appropriate adjustment of both the electrode location and intensity of electrical stimulation was made in order to induce a stable and precise SSSEP.

\section{Experimental Procedure}

Experimental tasks were divided into modeling and interference tasks. There were two kinds of modeling tasks, the target task (T-Task) of right hand MI and nontarget task (N-Task) of rest. In MI-BCI, data from two tasks are typically used to construct a binary classifier. The motor intention of the target limb is called the target task, and the one used to build a classifier with the target task is called the nontarget task. In MI-BCI used for post-stroke rehabilitation, the target task is generally specified as the affected limb MI (in this paper, the right hand is assumed to be the affected limb), and the nontarget task is specified as rest. During the rehabilitation treatment, when the classifier judges that the user is performing the target task, it will drive the peripheral equipment to pull the affected limb resulting in passive movement. This process can be considered as one trigger.

Three interference tasks (I-Task) were designed in this study to analyze the false triggering in MI-BCI, including left hand MI (I-Task1), left hand ME (I-Task2), and mental arithmetic (I- 
Task3) task. Interference tasks were not included in the modeling process but were used to test the built model. The ideal MI-BCI should be triggered only by the target task, and should not be triggered by any other mental activity that was not a target task. Hence, if MI-BCI is triggered by an interference task, this phenomenon will be considered as a false trigger.

Each task is described as follows: T-Task requires subjects to perform right hand MI, simulating rehabilitation treatment of the right hand. N-Task requires subjects to remain calm without any mental activity. For the three interference tasks, I-Task1 requires subjects to perform left hand $\mathrm{MI}$, simulating possible contralateral limb MI. I-Task2 requires subjects to perform left hand $\mathrm{ME}$ to simulate possible contralateral limb ME. The first two interference tasks are typical contralateral compensatory activities in stroke patients. I-Task3 involves subjects adding two random three-digit numbers displayed on the screen in their brains. This task simulates mental activities that have a specific workload but are irrelevant to the movement. Fig. 1(a) shows the fist state during hand MI or ME period, and the relax state during the non-hand task or non-task period.

The task display interface was developed using the Psychtoolbox-3 toolbox for MATLAB. The experimental process for collecting data on each task is defined as a trial, and the total duration of each trial was 10 seconds. At the beginning of each trial, a white circle lit up on the center of the screen for 2 seconds, indicating the start of a new trial. Next, the white circle disappeared, and a red circle appeared that lasted for 2 seconds (to remind subjects that the task was about to begin). The task prompt period lasted for 4 seconds, with the text (right hand $\mathrm{MI} /$ rest/left hand $\mathrm{MI} / \mathrm{left}$ hand $\mathrm{ME} /$ mental arithmetic) displayed on the center of the screen. This period required the subjects to perform the corresponding tasks according to the prompts, followed by 2 seconds, and then the subjects entered a relaxed state. The structure of a single trial is shown in fig. 1(b). In the hybrid paradigm, electrical stimulation was applied to the right median nerve when the red circle lit up and reached a maximum after 0.5 seconds. In each trial, maximum stimulation was between the 2nd and 8th second. During the period of the task prompt, subjects performed the corresponding task while simultaneously receiving the electrical stimulation. Fig. 1(c) shows the workflow of electrical stimulation.

The whole experiment was divided into 8 randomly-ordered sessions ( 4 sessions for the MI paradigm and 4 sessions for the hybrid paradigm). Each session contained 20 modeling trials (10 trials each for T-Task and N-Task) and 15 interference trials (5 trials each for I-Task1, I-Task2, and I-Task3). The individual paradigms had a total of 140 trials (40 trials each for T-Task and N-Task, and 20 trials each for I-Task1, I-Task2, and ITask3). There was a rest period of 2-5 minutes between each session to avoid fatigue during the experiment.

\section{Data Acquisition and Preprocessing}

The Neuroscan SynAmps2 system was used to acquire EEG data with 60 channels according to the international 10/20 system [27]. The ground electrode was placed on the forehead, and the reference electrode was placed on the nose. EEG data were band-pass filtered between 0.5 and $100 \mathrm{~Hz}$ with a sampling frequency of $1000 \mathrm{~Hz}$. An additional $50 \mathrm{~Hz}$ notch filter was used to remove power frequency interference during data acquisition. In the preprocessing stage, the raw data were spatially filtered using the common average reference (CAR) and then downsampled at $200 \mathrm{~Hz}$.

\section{Event-Related Spectral Perturbation}

ERD and SSSEP features were quantified by Event-related Spectral Perturbation (ERSP). ERSP was calculated by Shorttime Fourier Transform (STFT) with a Hanning-tapered window. Furthermore, to get the baseline-normalized ERSP, the mean spectrum in a baseline period ( $2 \mathrm{~s}$ before applying electrical stimulation) was subtracted from the original spectral estimation [28]. The ERSP value of ERD/SSSEP features could be calculated according to equation (1):

$$
E R D / S S S E P=\frac{1}{N} \sum_{f \in F} \sum_{t \in T}(\operatorname{ERSP}(f, t))
$$

The frequency of electrical stimulation was $31 \mathrm{~Hz}$ in the experiment, so $30-32 \mathrm{~Hz}$ was selected as the frequency band of SSSEP. In equation (1), F represents the frequency band of ERD or SSSEP, T represents the time interval of task execution, and $\mathrm{N}$ is the number of all the sampling points in the specific frequency band and time interval.

\section{Feature and Classifier}

In this study, we evaluated the recognition performance under four conditions, namely M-E (ERD in the MI paradigm), H-E (ERD in the hybrid paradigm), H-S (SSSEP in the hybrid paradigm) and H-ES (ERD and SSSEP in the hybrid paradigm). Taking H-E as an example, it denotes that ERD features are selected for classification in the hybrid paradigm. The corresponding features under the four different conditions are shown in Table 1 , where the symbol $(\sqrt{ })$ represents the features used in a specific condition. The M-E and H-E were compared so that we could determine whether the induced SSSEP under hybrid paradigm will affect the existing ERD feature. H-S was used to analyze the specificity of the SSSEP feature. Moreover, $\mathrm{M}-\mathrm{E}$ and H-ES were compared to verify whether the proposed hybrid paradigm could improve the performance of the MI-BCI system.

In this study, the CSP algorithm was used to extract EEG features [29], and SVM was used to build a classifier [30]. For each condition, the data in the task prompt period of each trial were selected for processing. First, the bandpass filter was used to extract corresponding frequency band of different features. Then for each frequency band, the CSP algorithm was used for spatial filtering where the first $\mathrm{N}$ features were selected. Finally, the feature vector used for recognition was the combination of these features in each frequency band. Target and nontarget task data were used to build a linear SVM classifier. 


\section{Evaluation Index}

In this study, the recognition performance is evaluated using three indexes, which are the $\mathrm{T}$-Task/N-Task recognition rate, false triggering rate, and AUC value. The predicted results of each task were abbreviated, to facilitate expression, as shown in Table 2. TT (True Target) represents the number of trials whose predicted label is the target task, and the predicted result is true. FN (False Nontarget) represents the number of trials whose predicted label is the nontarget task, and the predicted result is false. And other abbreviations can be deduced by analogy.

The first index is the T-Task/N-Task recognition rate. It is necessary to compare the classification performance of the target and nontarget task under four conditions. For each condition, the modeling trials were divided into training and testing sets through a tenfold cross-validation strategy. The kth $\mathrm{T}$-Task/N-Task recognition rate was obtained according to equation (2). Then the recognition rate for this condition was computed, as shown in equation (3).

$$
\begin{gathered}
a c c^{(k)}=\frac{T T+T N}{T T+F T+F N+T N}(k=1,2, \ldots, 10) \\
a c c_{\text {ave }}=\frac{1}{10} \sum_{k=1}^{10} a c c^{(k)}
\end{gathered}
$$

The second index is the false triggering rate of I-Task1, ITask2, and I-Task3 under four conditions. Modeling trials were used to build a T-Task/N-Task classifier, and then three types of interference trials (I-Task1, I-Task2, and I-Task3) were put into the classifier respectively to record the number of trials identified as T-Task $\left(F T_{i}\right)$. The proportion of $F T_{i}$ in the total number of such type $\left(F T_{i}+T N_{i}\right)$ was called the false triggering rate $\mathrm{fal}_{i}$ (as shown in equation (4)). The average false triggering rate $f a l_{\text {ave }}$ was obtained by equation (5).

$$
\begin{gathered}
f a l_{i}=\frac{F T_{i}}{F T_{i}+T N_{i}}(i=1,2,3) \\
\text { fal }_{\text {ave }}=\frac{1}{3} \sum_{i=1}^{3} f a l_{i}
\end{gathered}
$$

AUC, the area under the curve of Receiver Operating Characteristic (ROC), is used to evaluate the overall performance of the classifier [31]. Take T-Task as positive examples and other tasks (N-Task, I-Task1, I-Task2, and ITask3) as negative examples. True Positive Rate (TPR) is defined as the proportion of the trials that are predicted to be positive in trials that are actually positive, as shown in equation (6). False Positive Rate (FPR) is defined as the proportion of the trials that are predicted to be positive in trials that are actually negative, as shown in equation (7). For 140 trials under each paradigm, a series of coordinates (FPR, TPR) were obtained to calculate AUC values.

$$
\begin{gathered}
T P R=\frac{T T}{T T+F N} \\
F P R=\frac{F T+\sum_{i=1}^{3} F T_{i}}{F T+\sum_{i=1}^{3} F T_{i}+T N+\sum_{i=1}^{3} T N_{i}}
\end{gathered}
$$

\section{Specificity Analysis}

Low task specificity of ERD in the MI paradigm is the primary cause of false triggering. Therefore, the performance of the hybrid paradigm to reduce this phenomenon will depend on whether SSSEP modulated by MI has better task specificity, that is, whether there is better separability between T-Task and I-Task. Therefore, two paradigms need to be analyzed on the feature level. Sensitivity and specificity were defined as the evaluation indexes of the features, and they were obtained by equation (8) and (9), respectively.

The alpha and beta feature in the MI paradigm and the SSSEP feature in the MI-SSSEP paradigm at electrode C3 was used to build a Naive Bayes classifier [32] to calculate the feature sensitivity and specificity, respectively.

$$
\begin{gathered}
\text { sensitivity }=\frac{T T}{T T+F N} \\
\text { specificity }=\frac{T N+\sum_{i=1}^{3} T N_{i}}{F T+\sum_{i=1}^{3} F T_{i}+T N+\sum_{i=1}^{3} T N_{i}}
\end{gathered}
$$

\section{Results}

Fig. 2 presents the average T-Task/N-Task recognition rate, false triggering rate, and AUC value across 13 subjects under four conditions of M-E, H-ES, H-E, and H-S. The comparison shows that the $\mathrm{T}$-Task/N-Task recognition rate obtained under $\mathrm{H}-\mathrm{E}$ is similar to that under M-E $(\mathrm{p}=0.380)$, indicating that SSSEP induced in the hybrid paradigm does not affect the separability of the existing ERD feature. Additionally, the recognition rate in $\mathrm{H}-\mathrm{S}$ can reach the level of that in M-E. Moreover, there is no significant difference between the two $(\mathrm{p}=0.462)$, which indicates that the SSSEP feature could distinguish the target task from the nontarget task well. Furthermore, compared with the M-E condition, the recognition rate under H-ES increases significantly from $74.2 \%$ to $82.0 \%$ $(\mathrm{p}<0.01)$, illustrating that the SSSEP in the MI-SSSEP paradigm could improve the feature information, and thus, enhance the performance of classification.

Under the condition of M-E, all the interference tasks have high false triggering rates, reaching an average of $55.5 \%$. This indicates that many interference tasks could cause false triggering in $\mathrm{MI}-\mathrm{BCI}$, which is a significant problem for MIBCI applications. While for the H-S condition using SSSEP, the false triggering rate of three interference tasks decreases significantly. Specifically, the I-Task1 decreases from $65.8 \%$ to $33.4 \%$ ( $\mathrm{p}<0.001$ ), the I-Task 2 decreases from $61.5 \%$ to $35.7 \%$ $(\mathrm{p}<0.001)$, the I-Task3 decreases from $39.2 \%$ to $18.8 \%$ $(\mathrm{p}<0.05)$, and the average false triggering rate decreases from $55.5 \%$ to $29.3 \% \quad(\mathrm{p}<0.001)$. This result validates a key assumption of our research study that the SSSEP feature in the MI-SSSEP paradigm has better task specificity. It has both the almost equivalent T-Task/N-Task separability and better task specificity compared with ERD so that the T-Task/N-Task classifier is not easily triggered when the brain performs interference tasks.

The AUC value is used as another evaluation metric to assess the overall performance of the classifier. Compared with the ME condition, the AUC value under H-ES significantly increases 
from 0.669 to 0.788 ( $\mathrm{p}<0.01)$. Meanwhile, the AUC value under $\mathrm{H}-\mathrm{S}$ also increases to 0.771 , indicating that SSSEP in the hybrid paradigm improves the overall performance of MI-BCI.

In order to compare the difference between the induced features under the MI and MI-SSSEP paradigm, the timefrequency maps of the target task, nontarget task, and interference tasks at electrode C3 are shown in Fig. 3. The red dotted line represents the start time of electrical stimulation in the hybrid paradigm, the blue dotted line represents the start of task execution, and the black dotted line represents the end of task execution or the time when the electrical stimulation disappears. Under the MI paradigm, it could be seen that, in line with our expectation, the target task induced long-term ERD at electrode C3. However, interference tasks also produced ERD, though it was relatively weaker than that in the target task, it still made it difficult for classification in the MI-BCI system. Moreover, under the MI-SSSEP paradigm, SSSEP coexisted with the ERD feature, and the ERD induced by N-Task and ITask was mainly due to somatosensory response. It can be seen that SSSEP was induced at the same frequency from the beginning to the end of the electrical stimulation, indicating that the SSSEP feature was successfully induced by external stimulation.

The ERSP curves of each feature at electrode C3 in the MI and MI-SSSEP paradigm are shown in Fig. 4. The blue curve represents the target task, the red curve represents the nontarget task, and the yellow curve represents the average interference task. For alpha and beta features under the two paradigms, there was an energy decline on both the target and interference task during task execution. It can be seen that the interference task could induce ERD features similar to the target task, though the intensity was somewhat weaker. This phenomenon partly explains why the classifier mistakenly identifies some interference tasks as target tasks. For the SSSEP feature in the hybrid paradigm, when affected by the target task MI, there will be a significant energy drop during the task execution. At the same time, there is no such energy change in the interference task. This finding indicates that the interference task does not affect SSSEP responses in EEG. In terms of features, the interference task does not produce similar features compared with the target task. Therefore, the feature of SSSEP modulated by MI shows fine task specificity, which makes the false triggering in MI-BCI well suppressed.

The sensitivity and specificity histograms of the alpha and beta feature in the MI paradigm and SSSEP feature in the MISSSEP paradigm are shown in Fig. 5. For the specificity index, the SSSEP feature in the MI-SSSEP paradigm reaches 0.678, which is significantly higher than the alpha feature by 0.289 $(\mathrm{p}<0.001)$ and the beta feature by $0.167(\mathrm{p}<0.05)$ in MI paradigm. The result indicates that the SSSEP feature could reduce the probability of identifying the nontarget or interference task as a target task. It is consistent with the result that the H-S condition could significantly reduce the false triggering rate (Fig. 2). For the sensitivity index, although the SSSEP feature is slightly lower than ERD, it still shows a strong ability to distinguish the target task from other tasks. Therefore, analysis on the feature level validates the assumption that
SSSEP modulated by MI has better task specificity.

\section{Discussion}

To our knowledge, in the field of MI-BCI, very few related studies have addressed the influence of irrelevant mental activities on classification and proposed methods for reducing false triggering. To this end, we have carried out experimental studies to explore the causes of false triggering and the methods that can reduce the phenomenon. Experimental results demonstrate that false triggering does exist in MI-BCI systems, which may result in a wrong neural induction effect and thus promote irreversible harm during post-stroke rehabilitation. Therefore, the problem of false triggering (and its suppression) is indeed worthy of attention in clinical applications.

SSSEP is a feature induced by sensation, while MI is a sense of movement, which means that the modulation of SSSEP by MI may have a more complicated mechanism. One possible mechanism is the resource competition between motor intention and sensory awareness because both of them involve the primary sensorimotor cortex [33], [34], which may cause MI to suppress SSSEP [35]. Specifically, when somatosensory stimulation at a fixed frequency is applied to the target limb, the SSSEP of the corresponding frequency will be induced in the sensorimotor cortex. Therefore, when subjects are receiving electrical stimulation while performing MI on the same limb, some of the original neurons used to generate SSSEP may be forced to process this MI task, thereby changing the existing SSSEP feature. Since somatosensory stimulation directly acts on the specific target limb, irrelevant mental activities are less likely to cause changes on SSSEP than the motor intention of this limb, so SSSEP modulated by MI feature may have better task specificity. Another possible mechanism may be related to attention. As a result of the presence of electrical stimulation, subjects may focus their attention on the corresponding limb during the experiment, thus affecting the steady-state somatosensory response [36].

In this study, only the right hand MI was used as the target task. In theory, left and right hand MI should be performed as the target task respectively in the experiment. However, it is costly to do such an experiment, and few studies have shown that there is a difference in the performance of right/left hand MI. Also, the sensory pathway may be impaired in stroke patients, so whether SSSEP could be induced and whether MI has a similar modulation effect on SSSEP compared with healthy people remains to be studied experimentally. Of course, many studies have shown that sensory awareness is retained in some stroke patients and is gradually restored during the process of stroke rehabilitation [37].

In future studies, more types of irrelevant tasks will be included to test the feasibility of the hybrid paradigm. Additionally, clinical experiments should be carried out to evaluate the efficacy of the new paradigm. The current CSP algorithm is not the optimal algorithm for narrow-band signals such as SSSEP, so a suitable signal processing algorithm needs to be introduced to improve the recognition performance. 


\section{Conclusions}

The ERD feature that MI-BCI decoding relies on has poor task specificity, which can easily cause false triggering in the system. Therefore, to reduce this phenomenon, the feature of SSSEP modulated by MI under the MI-SSSEP paradigm is discussed in this paper. Experimental research shows that the SSSEP feature in the hybrid paradigm has better task specificity than ERD, which can effectively reduce false triggering and improve the recognition performance of MI$\mathrm{BCI}$. This finding may provide a new way to enhance the overall performance of MI-BCI used for post-stroke rehabilitation.

\section{Abbreviations \\ BCI: brain-computer interface; MI: motor imagery; ERD: event-related desynchronization; SSSEP: steady-state somatosensory evoked potential; MI-SSSEP: the SSSEP is combined with the MI; EEG: electroencephalography; SCI: spinal cord injury; ALS: amyotrophic lateral sclerosis; ME: motor execution; CSP: common spatial pattern; SVM: support vector machine; T-Task: target task; N-Task: nontarget task; I- Task: interference tasks; I-Task1: left hand MI; I-Task2: left hand ME; I-Task3: mental arithmetic; CAR: common average reference; ERSP: Event-related Spectral Perturbation; STFT: Short-time Fourier Transform; M-E: ERD in the MI paradigm; H-E: ERD in the hybrid paradigm; H-S: SSSEP in the hybrid paradigm; H-ES: ERD and SSSEP in the hybrid paradigm; TT: True Target; FN: False Nontarget; ROC: Receiver Operating Characteristic; AUC: the area under the curve of ROC; TPR: True Positive Rate; FPR: False Positive Rate.}

\section{Ethics approval and consent to participate}

Before the formal experiment, all subjects were informed about the specific experimental procedure and signed their informed consent. This study was approved by the Department of Biomedical Engineering of Tianjin University and the ethical committee of Tianjin University.

\section{Consent for publication}

The subjects consent with publication of their data.

\section{Availability of data and materials}

Please contact author for data requests.

\section{Competing Interests}

The authors declare that they have no competing interests.

\section{Funding}

This work was supported by National Natural Science Foundation of China (No.91648122) and Natural Science Foundation of Tianjin City (No.19JCYBJC28700).

\section{Authors' contributions}

LZ and HQ designed the method and drafted the manuscript. $\mathrm{LZ}$ and XT carried out the data acquisition and analysis. FH and PZ supervised the study, helped revise the manuscript. All authors read and approved the final manuscript.

\section{Acknowledgments}

Not applicable.

\section{References}

1. N. Padfield, J. Zabalza, H. M. Zhao, V. Masero, and J. C. Ren. EEGBased Brain-Computer Interfaces Using Motor-Imagery: Techniques and Challenges. Sensors 2019; 19(6): 1423-56.

2. R. Leeb, D. Friedman, G. R. Muller-Putz, R. Scherer, M. Slater, and G. Pfurtscheller. Self-paced (asynchronous) BCI control of a wheelchair in virtual environments: a case study with a tetraplegic. Comput. Intell. Neurosci. 2007; 2007: 79642-9.

3. A. Kubler, F. Nijboer, J. Mellinger, T. M. Vaughan, H. Pawelzik, G. Schalk, D. J. McFarland, N. Birbaumer, and J. R. Wolpaw. Patients with ALS can use sensorimotor rhythms to operate a brain-computer interface. Neurology. 2005; 64(10): 1775-7.

4. A. Ramos-Murguialday, D. Broetz, M. Rea, L. Laer, O. Yilmaz, F. L. Brasil, G. Liberati, M. R. Curado, E. Garcia-Cossio, A. Vyziotis, W. Cho, M. Agostini, E. Soares, S. Soekadar, A. Caria, L. G. Cohen, and N. Birbaumer. Brain-machine interface in chronic stroke rehabilitation: a controlled study. Ann. Neurol. 2013; 74(1): 100-8.

5. G. Prasad, P. Herman, D. Coyle, S. McDonough, and J. Crosbie. Applying a brain-computer interface to support motor imagery practice in people with stroke for upper limb recovery: a feasibility study. $J$. Neuroeng. Rehabil 2010; 7(1): 60-76.

6. A. Remsik, B. Young, R. Vermilyea, L. Kiekhoefer, J. Abrams, S. Evander Elmore, P. Schultz, V. Nair, D. Edwards, J. Williams, and V. Prabhakaran. A review of the progression and future implications of brain-computer interface therapies for restoration of distal upper extremity motor function after stroke. Expert Rev. Med. Devices 2016; 13(5): 445-54

7. A. Biasiucci, R. Leeb, I. Iturrate, S. Perdikis, A. Al-Khodairy, T. Corbet, A. Schnider, T. Schmidlin, H. Zhang, M. Bassolino, D. Viceic, P. Vuadens, A. G. Guggisberg, and J. D. R. Millan. Brain-actuated functional electrical stimulation elicits lasting arm motor recovery after stroke. Nat. Commun 2018; 9(1): 2421-34.

8. B. Blankertz, R. Tomioka, S. Lemm, M. Kawanabe, and K. R. Muller. Optimizing spatial filters for robust EEG single-trial analysis. IEEE Signal Process Mag 2008; 25(1): 41-56.

9. M. Jeannerod. Neural simulation of action: a unifying mechanism for motor cognition. Neuroimage 2001; 14(1 Pt 2): S103-9.

10. A. J. Szameitat, S. Shen, and A. Sterr. Motor imagery of complex everyday movements. An fMRI study. Neuroimage 2007; 34(2): 702-13.

11. Z. Tang, S. Sun, S. Zhang, Y. Chen, C. Li, and S. Chen. A Brain-Machine Interface Based on ERD/ERS for an Upper-Limb Exoskeleton Control. Sensors (Basel) 2016; 16(12): 2050-63.

12. R. A. Ramadan, and A. V. Vasilakos. Brain computer interface: control signals review. Neurocomputing 2017; 223: 26-44.

13. B. Z. Allison, E. W. Wolpaw, and J. R. Wolpaw. Brain-computer interface systems: progress and prospects. Expert Rev. Med. Devices 2007; 4(4): 463-74.

14. G. Pfurtscheller, C. Brunner, A. Schlogl, and F. H. Lopes da Silva. Mu rhythm (de)synchronization and EEG single-trial classification of different motor imagery tasks. Neuroimage 2006; 31(1): 153-9.

15. G. Pfurtscheller, and F. H. Lopes da Silva. Event-related EEG/MEG synchronization and desynchronization: basic principles. Clin. Neurophysiol. 1999; 110(11): 1842-57.

16. M. Z. Baig, N. Aslam, H. P. H. Shum, and L. Zhang. Differential evolution algorithm as a tool for optimal feature subset selection in motor imagery EEG. Expert Syst. Appl. 2017; 90: 184-95.

17. A. Subasi, and M. I. Gursoy. EEG signal classification using PCA, ICA, LDA and support vector machines. Expert Syst. Appl. 2010; 37(12): 8659-66.

18. M. Grosse-Wentrup, D. Mattia, and K. Oweiss. Using brain-computer interfaces to induce neural plasticity and restore function. J. Neural Eng. 2011; 8(2): 025004-10.

19. A. Jackson, and J. B. Zimmermann. Neural interfaces for the brain and spinal cord-restoring motor function. Nat. Rev. Neurol. 2012; 8(12): 6909.

20. P. Zhuang, C. Toro, J. Grafman, P. Manganotti, L. Leocani, and M. Hallett. Event-related desynchronization (ERD) in the alpha frequency 
during development of implicit and explicit learning. Electroencephalogr. Clin. Neurophysiol. 1997; 102(4): 374-81.

21. M. Hassan, O. Dufor, I. Merlet, C. Berrou, and F. Wendling. EEG source connectivity analysis: from dense array recordings to brain networks. PLoS One 2014; 9(8): e105041-55.

22. S. Ahn, K. Kim, and S. C. Jun. Steady-State Somatosensory Evoked Potential for Brain-Computer Interface-Present and Future. Front. Hum. Neurosci. 2015; 9: 716-21.

23. S. Ahn, M. Ahn, H. Cho, and S. Chan Jun. Achieving a hybrid braincomputer interface with tactile selective attention and motor imagery. $J$. Neural Eng. 2014; 11(6): 066004-17.

24. W. Yi, S. Qiu, K. Wang, H. Qi, X. Zhao, F. He, P. Zhou, J. Yang, and D. Ming. Enhancing performance of a motor imagery based brain-computer interface by incorporating electrical stimulation-induced SSSEP. $J$. Neural Eng. 2017; 14(2): 026002-11.

25. Z. Chen, Z. Wang, K. Wang, W. Yi, and H. J. I. A. Qi. Recognizing motor imagery between hand and forearm in the same limb in a hybrid brain computer interface paradigm: An online study. IEEE Access 2019; 7: 59631-9.

26. R. S. Noss, C. D. Boles, and C. D. Yingling. Steady-state analysis of somatosensory evoked potentials. Electroencephalogr. Clin. Neurophysiol. 1996; 100(5): 453-61.

27. G. H. Klem, H. O. Luders, H. H. Jasper, and C. Elger. The ten-twenty electrode system of the International Federation. The International Federation of Clinical Neurophysiology. Electroencephalogr. Clin. Neurophysiol. Suppl. 1999; 52: 3-6.

28. A. Delorme, and S. Makeig. EEGLAB: an open source toolbox for analysis of single-trial EEG dynamics including independent component analysis. J. Neurosci. Methods 2004; 134(1): 9-21.
29. H. Ramoser, J. Muller-Gerking, and G. Pfurtscheller. Optimal spatial filtering of single trial EEG during imagined hand movement. IEEE Trans. Rehabil. Eng. 2000; 8(4): 441-6.

30. C. C. Chang, and C. J. Lin. LIBSVM: A Library for Support Vector Machines. Acm Trans Intell Syst and Technol 2011; 2(3): 1-27.

31. A. P. Bradley. The use of the area under the ROC curve in the evaluation of machine learning algorithms. Pattern recognition 1997; 30(7): 114559.

32. I. Rish. An empirical study of the naive Bayes classifier. IJCAI 2001 workshop on empirical methods in artificial intelligence 2001; 3(22): 416.

33. M. Hallett, J. Fieldman, L. G. Cohen, N. Sadato, and A. Pascual-Leone. Involvement of primary motor cortex in motor imagery and mental practice. Behavioral and Brain Sciences 1994; 17(2): 187-245.

34. G. R. Muller-Putz, R. Scherer, C. Neuper, and G. Pfurtscheller. Steadystate somatosensory evoked potentials: suitable brain signals for braincomputer interfaces? IEEE Trans. Neural Syst. Rehabil. Eng. 2006; 14(1): 30-7.

35. S. A. Josselyn, and P. W. Frankland. Memory allocation: mechanisms and function. Annu. Rev. Neurosci. 2018; 41: 389-413.

36. S. R. Jones, C. E. Kerr, Q. Wan, D. L. Pritchett, M. Hamalainen, and C. I. Moore. Cued spatial attention drives functionally relevant modulation of the mu rhythm in primary somatosensory cortex. J. Neurosci. 2010; 30(41): 13760-5.

37. A. Caria, C. Weber, D. Brotz, A. Ramos, L. F. Ticini, A. Gharabaghi, C. Braun, and N. Birbaumer. Chronic stroke recovery after combined BCI training and physiotherapy: a case report. Psychophysiology 2011; 48(4): $578-82$. 


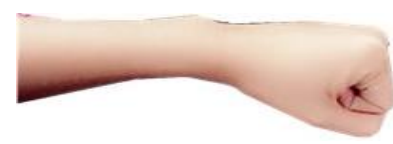

Make a fist

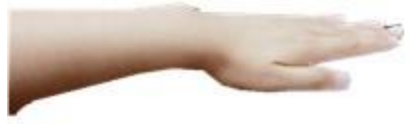

Relax

(a) Figure of hand action

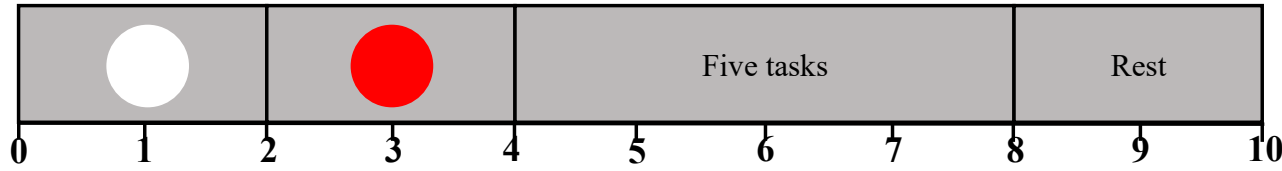

(b) The structure of a single trial

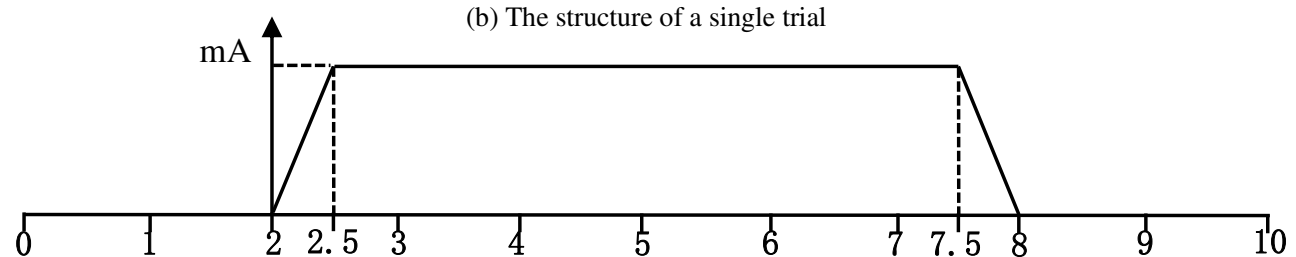

(c) The workflow for the electrical stimulation

Fig. 1 Experimental paradigm

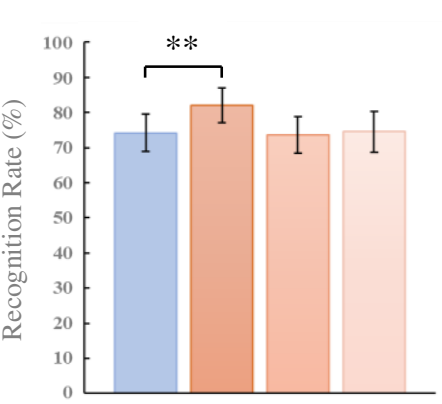

(a) T-Task / N-Task recognition rate

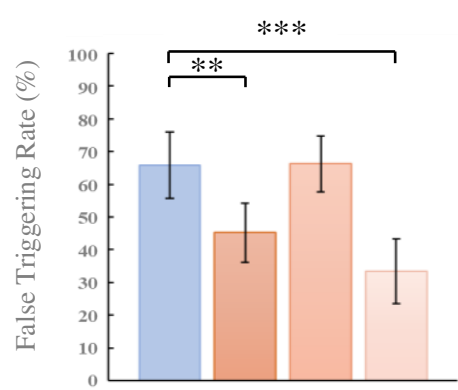

(d) False triggering rate of I-Task1

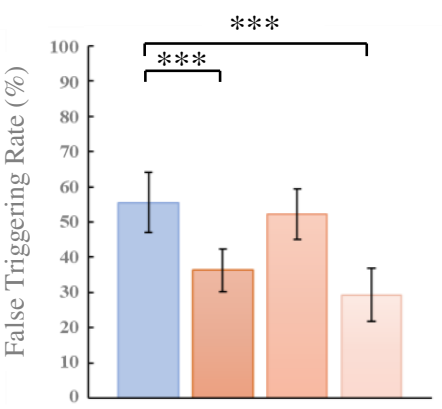

(b) Average false triggering rate of I-Task

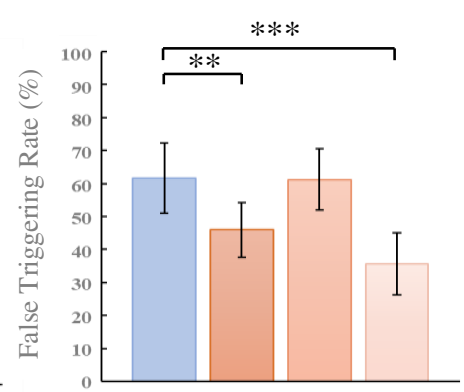

(e) False triggering rate of I-Task2

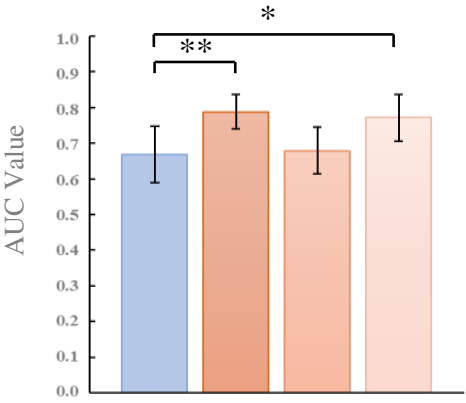

(c) AUC value

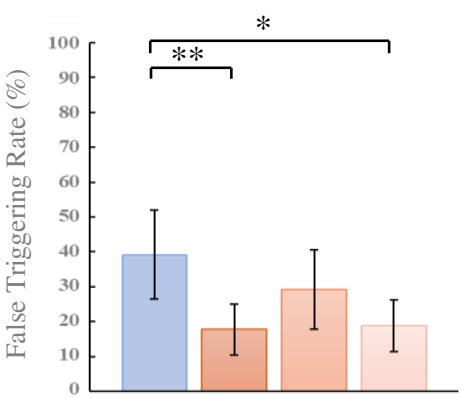

(f) False triggering rate of I-Task3

$\square$ M-E $\square$ H-ES $\square$ H-E $\square$ H-S

Fig. 2 The average evaluation indexes across 13 subjects under four conditions of M-E, H-ES, H-E, H-S. A significant difference through paired t-test is indicated by an asterisk $(\mathrm{p}<0.05)$ or two asterisks $(\mathrm{p}<0.01)$ or three asterisks $(\mathrm{p}<0.001)$. 

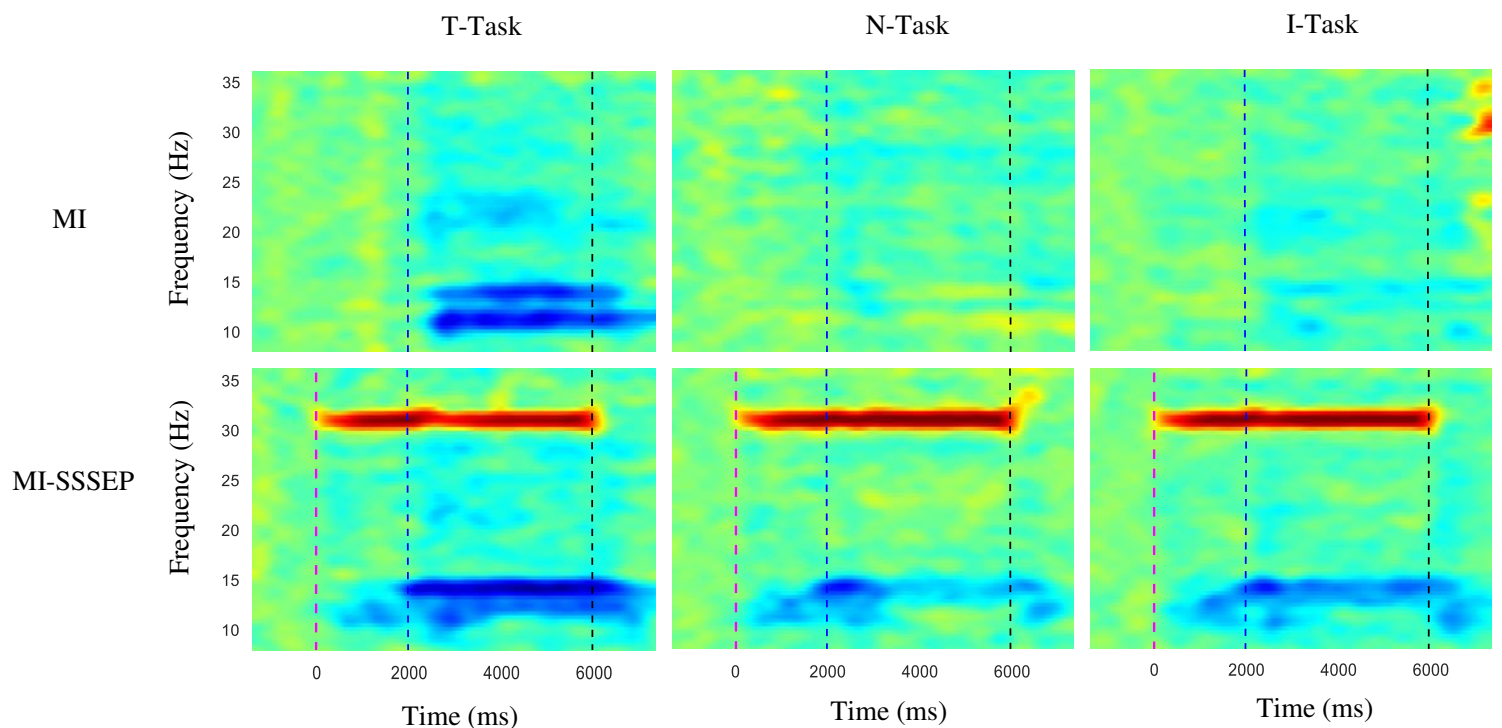

$\begin{array}{lllllllll}-8 & -6 & -4 & -2 & 0 & 2 & 4 & 6 & 8\end{array}$

ERSP (dB)

Fig. 3 Time-frequency maps of different tasks at electrode C3 under the MI and MI-SSSEP paradigm.
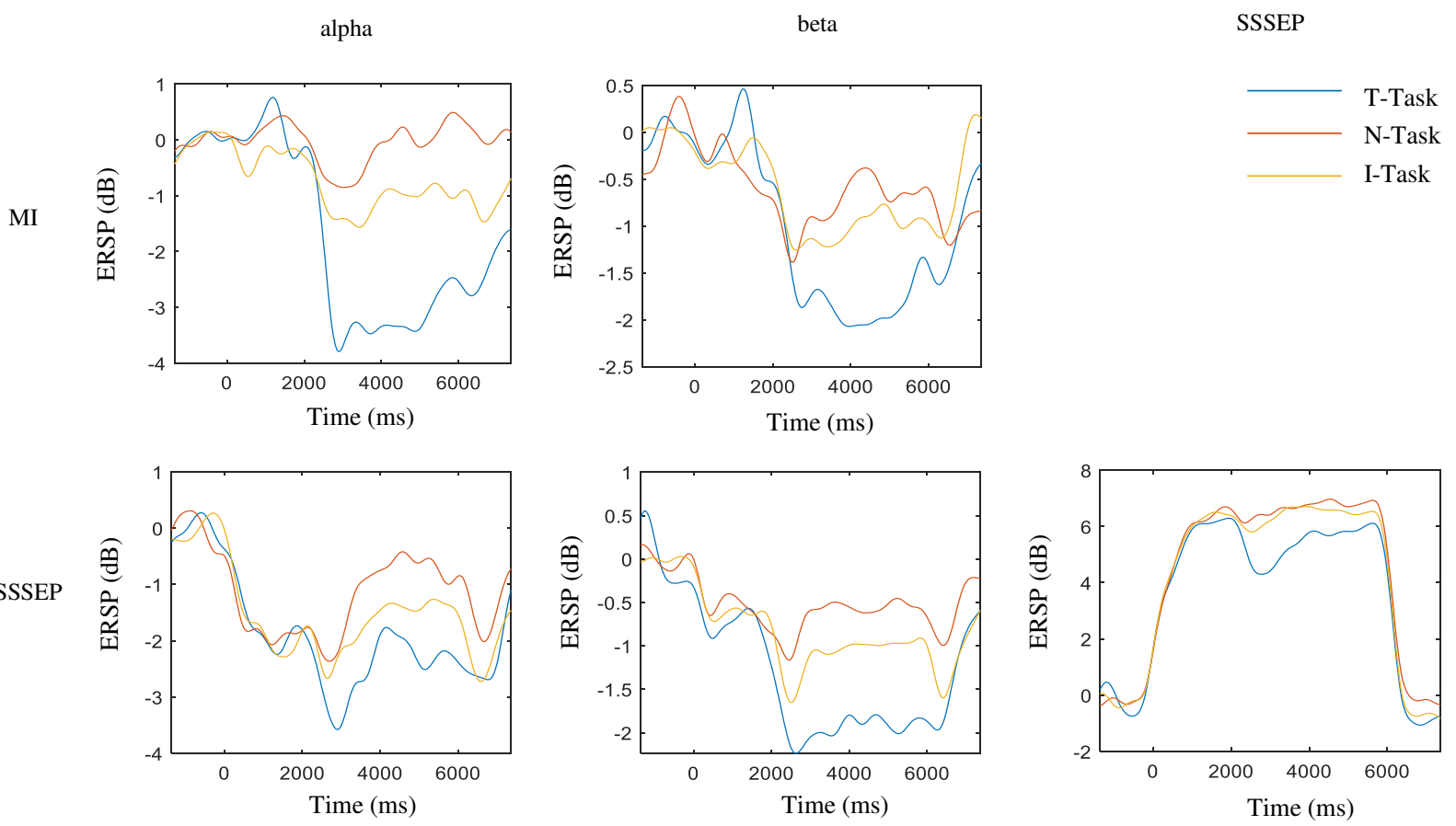

Fig. 4 ERSP curves of each feature at electrode C3 under the MI and MI-SSSEP paradigm. 


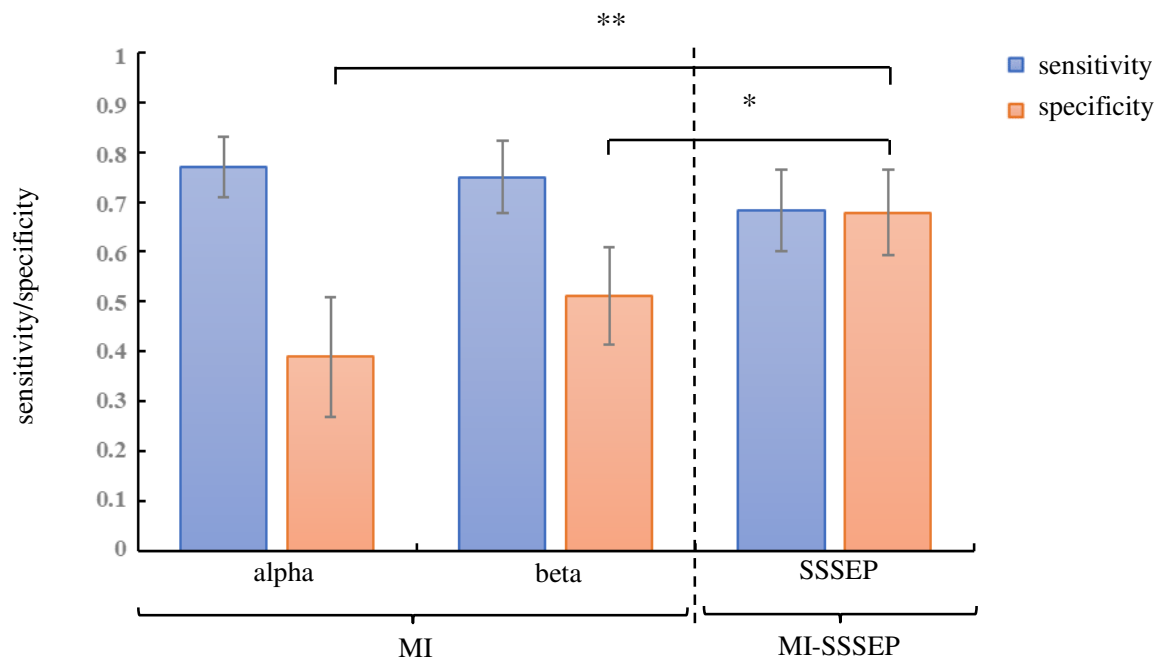

Fig. 5 Analysis of feature specificity at electrode $\mathbf{C} 3$ under two paradigms. A significant difference through paired t-test is indicated by an asterisk $(\mathrm{p}<0.05)$ or two asterisks $(\mathrm{p}<0.001)$.

Table 1 Corresponding Features Under Four Conditions

\begin{tabular}{cccccc}
\hline \multirow{2}{*}{ Predicted label } & \multicolumn{5}{c}{ True label } \\
\cline { 2 - 6 } & T-Task & N-Task & I-Task1 & I-Task2 & I-Task3 \\
\hline T-Task & TT & FT & $\mathrm{FT}_{1}$ & $\mathrm{FT}_{2}$ & $\mathrm{FT}_{3}$ \\
N-Task & FN & TN & $\mathrm{TN}_{1}$ & $\mathrm{TN}_{2}$ & $\mathrm{TN}_{3}$ \\
\hline
\end{tabular}

Table 2 Definition Of Predicted Results For Each Task

\begin{tabular}{ccccc}
\hline Feature & M-E & H-E & H-S & H-ES \\
\hline alpha $(8-13 \mathrm{~Hz})$ & $\sqrt{ }$ & $\sqrt{ }$ & & $\sqrt{ }$ \\
$\operatorname{beta}(13-30 \mathrm{~Hz})$ & $\sqrt{ }$ & $\sqrt{ }$ & & $\sqrt{ }$ \\
$\operatorname{SSSEP}(30-32 \mathrm{~Hz})$ & & & $\sqrt{ }$ & $\sqrt{ }$ \\
\hline
\end{tabular}


Figures

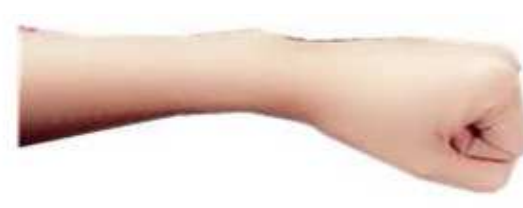

Make a fist

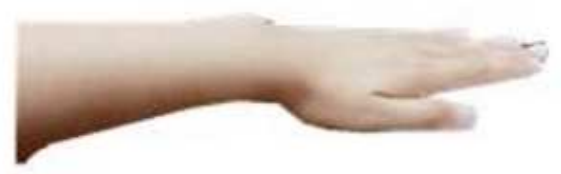

Relax

(a) Figure of hand action

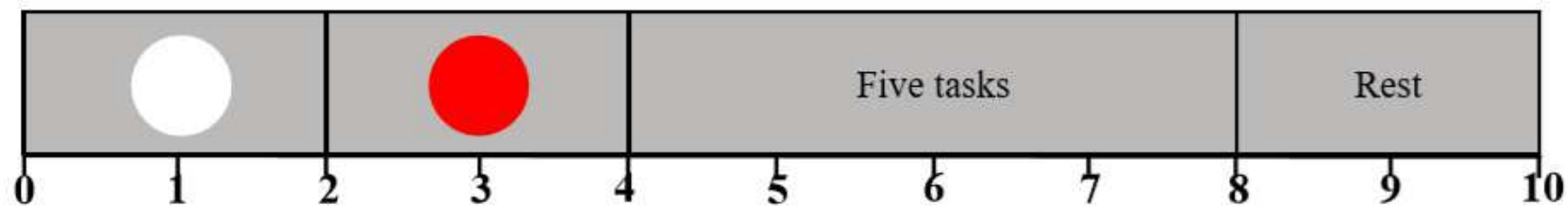

(b) The structure of a single trial

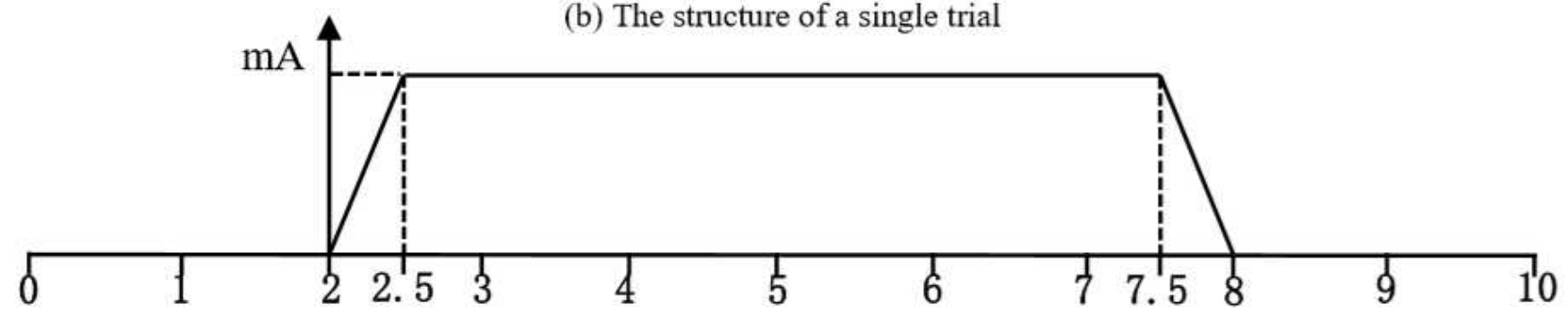

(c) The workflow for the electrical stimulation

Figure 1

Experimental paradigm 


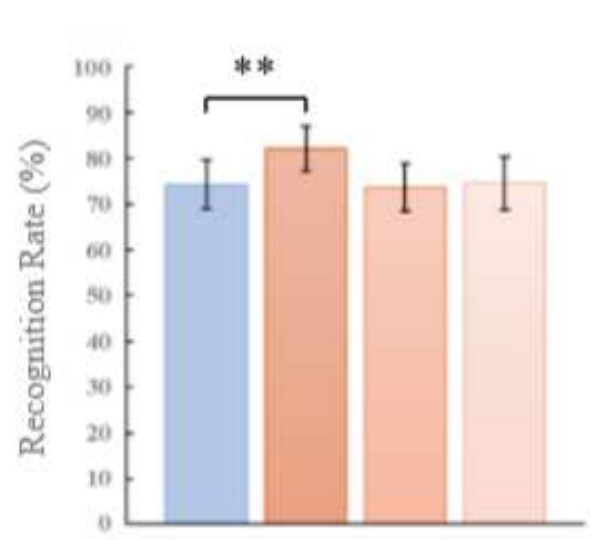

(a) T-Task / N-Task recognition rate

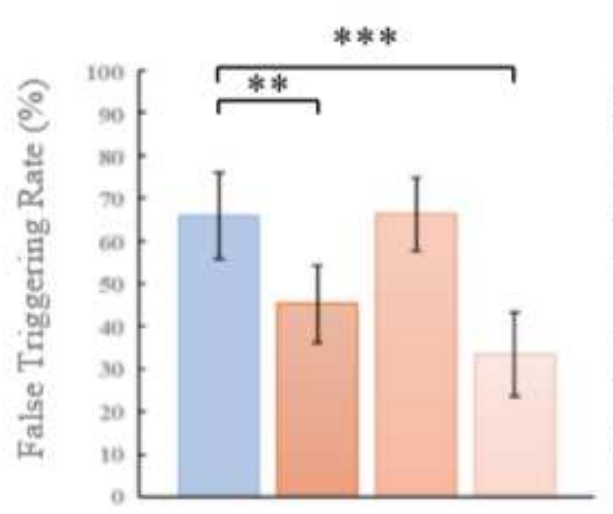

(d) False triggering rate of I-Task1

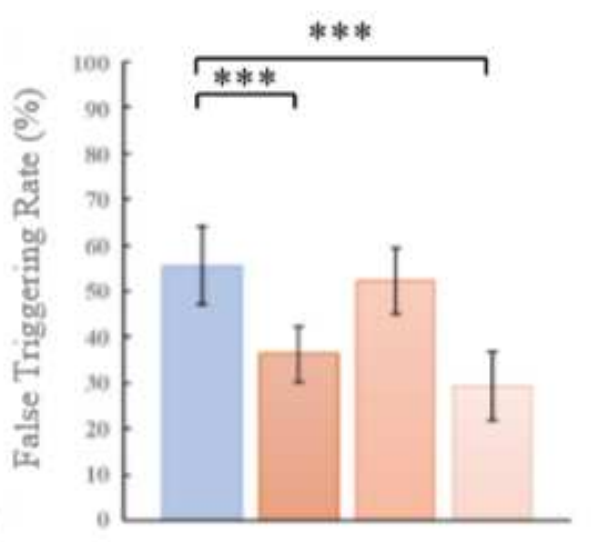

(b) Average false triggering rate of I-Task

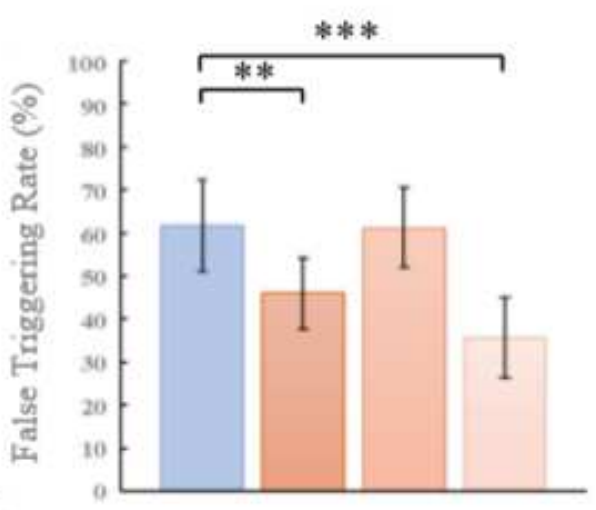

(e) False triggering rate of I-Task2

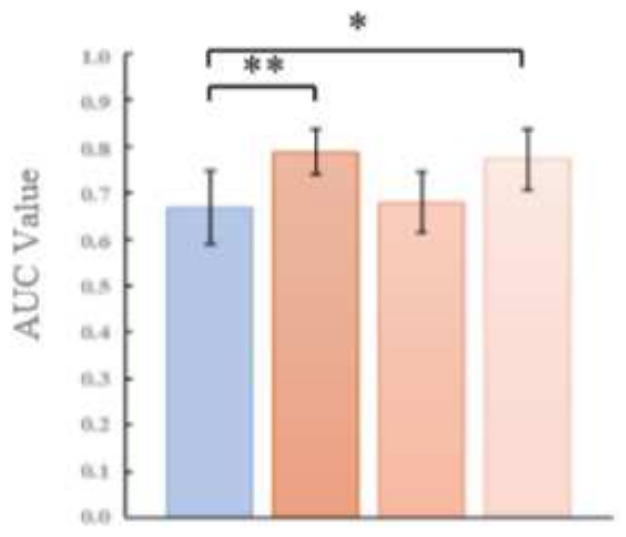

(c) AUC value

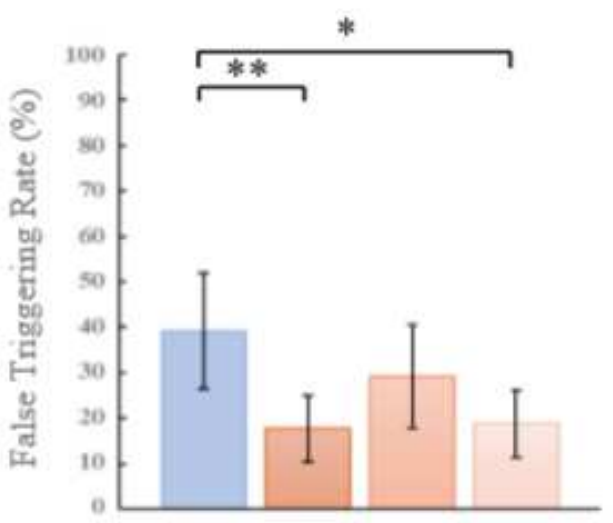

(f) False triggering rate of I-Task3

$\mathrm{M}-\mathrm{E} \square \mathrm{H}-\mathrm{ES} \square \mathrm{H}-\mathrm{E} \square \mathrm{H}-\mathrm{S}$

Figure 2

The average evaluation indexes across 13 subjects under four conditions of $M-E, H-E S, H-E, H-S$. A significant difference through paired $t$-test is indicated by an asterisk $(p<0.05)$ or two asterisks $(p<0.01)$ or three asterisks $(p<0.001)$. 
T-Task

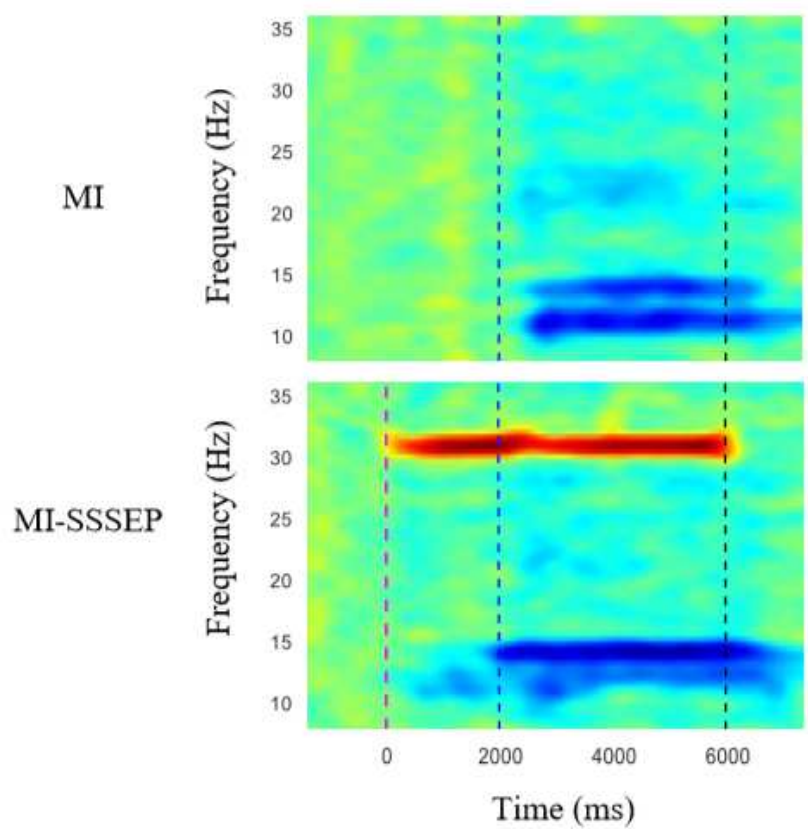

N-Task
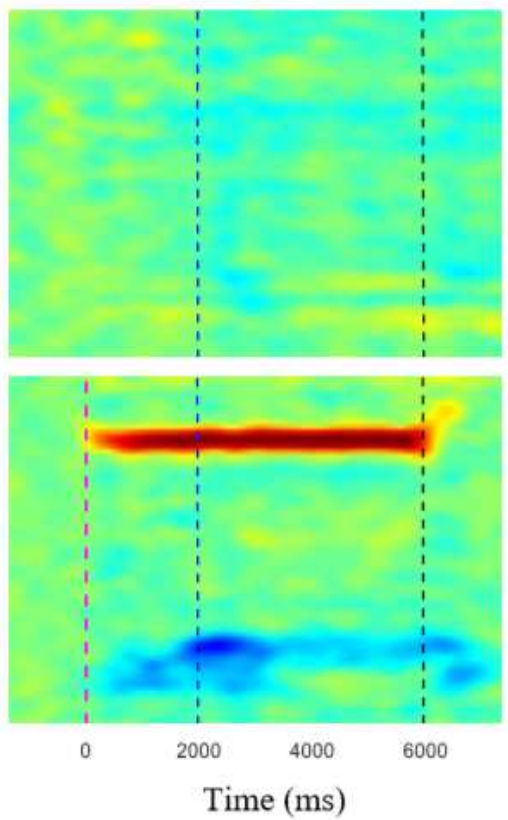

I-Task
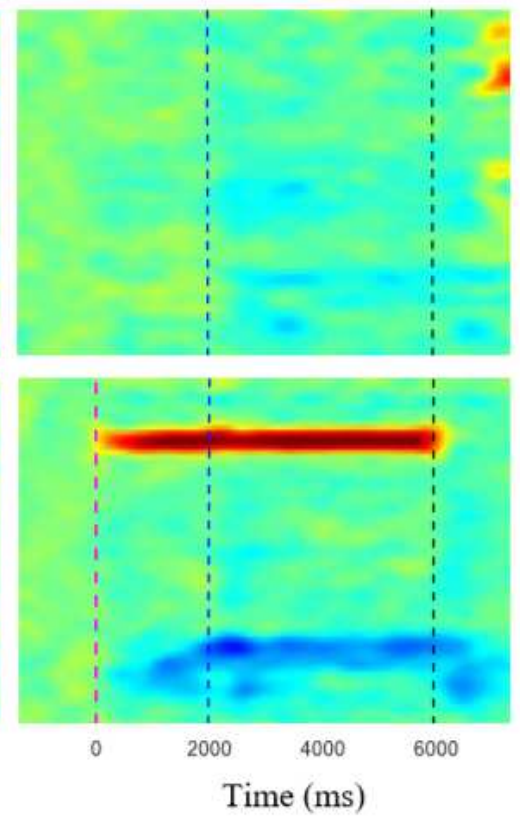

$$
\begin{array}{llllllllll}
-8 & -6 & -4 & -2 & 0 & 2 & 4 & 6 & 8 & \\
\hline & & & & & & & & &
\end{array}
$$

\section{Figure 3}

Time-frequency maps of different tasks at electrode C3 under the MI and MI-SSSEP paradigm. alpha beta SSSEP
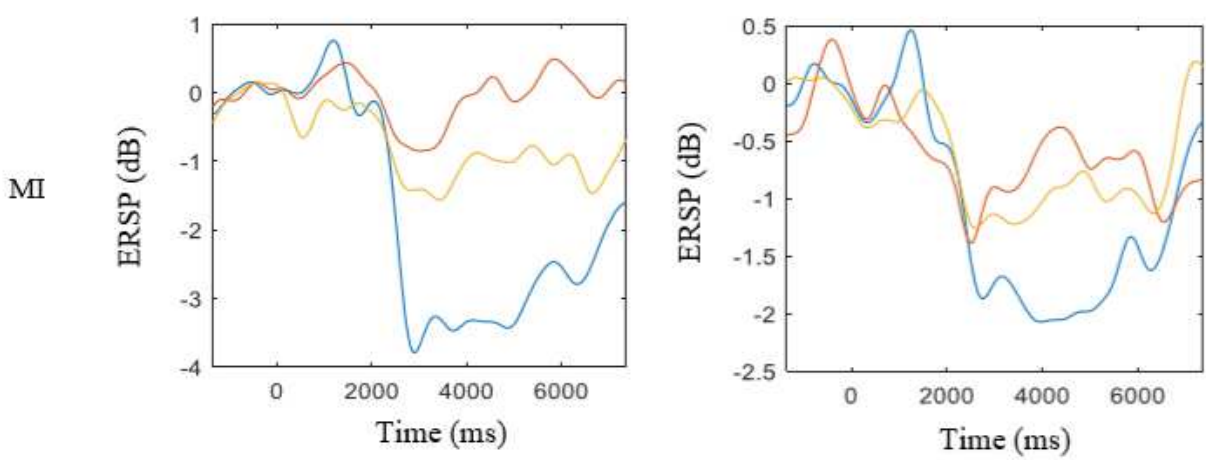

T-Task

N-Task

I-Task
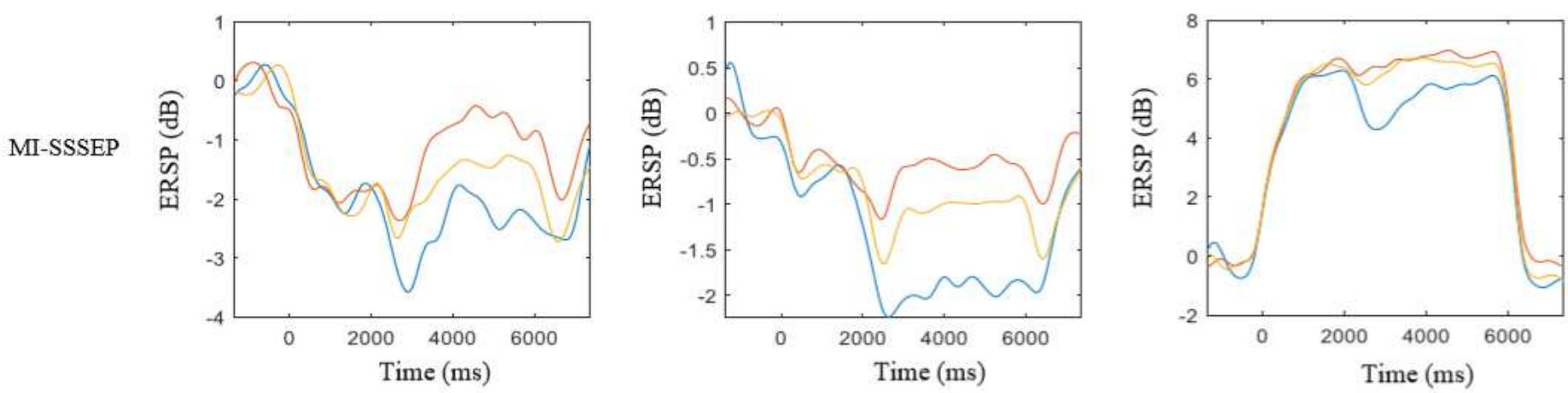

Figure 4 
ERSP curves of each feature at electrode $\mathrm{C} 3$ under the MI and MI-SSSEP paradigm.

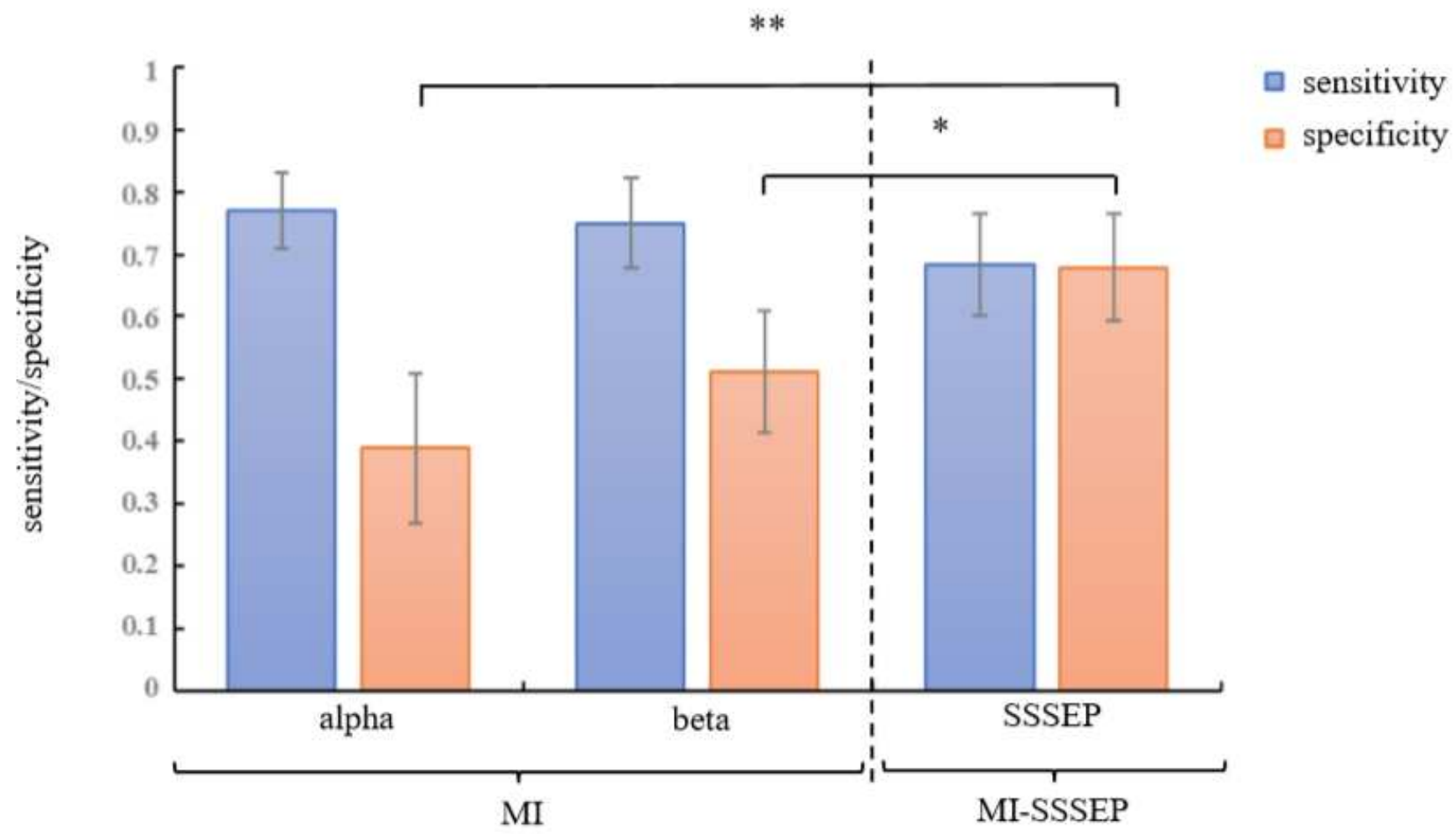

Figure 5

Analysis of feature specificity at electrode C3 under two paradigms. A significant difference through paired t-test is indicated by an asterisk $(p<0.05)$ or two asterisks $(p<0.001)$. 